\title{
International Journal \\ of \\ English Studies
}

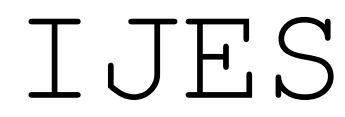

UNIVERSITY OF MURCIA

http://revistas.um.es/ijes

Review of Fuchs, Robert. 2016. Speech rhythm in varieties of English: Evidence from educated Indian English and British English. Singapore: Springer Science+Business Media Singapore. 226 pages. ISBN: 978-3-662-47818-9.

JANE SETTER

University of Reading

Fuchs (2016) is the monograph version of Robert Fuchs's PhD thesis (University of Münster, Germany, 2013). It details research carried out on the production and perception of speech rhythm in Indian English (IndE) and British English (BrE) among students at two Universities in Hyderabad, India, at the start of this decade, the development of "a multidimensional model of rhythm" (Fuchs, 2016: 4), and of an approach to speech rhythm perception research which modifies existing practice. From the outset, and just by reading the Preface to the volume (Fuchs, 2016: v-vii), one gets a sense of the enthusiasm Fuchs has for India, the people, the variety, and the research he has undertaken. This is a promising opening to what is basically a write-up of a research study, boding well for the rest of the book, and leading on to the introductory chapter in which the scene is set.

The book is organised into eight chapters, as follows:

a) Chapter 1 is a general introduction, outlining the available research at the time the study was undertaken and justifying the book's focus on speech rhythm in IndE, i.e., the lack of work on prosodic features of the variety. It also serves to situate the study firmly in a World Englishes paradigm, referring to Kachru's (1985) terminology of Inner, Outer and Expanding Circles, and Schneider's Dynamic Model of Postcolonial Englishes (2003, 2007).

b) Chapter 2 starts by giving a historical and social description of English in India, including an account of the different varieties of IndE and of the Dravidian and Aindo-Aryan languages spoken. It further elaborates on IndE with respect to Kachru and Schneider's models, before going on to give a comparison of the phonologies of BrE and IndE based on existing descriptions. 
c) Chapter 3's focus is on speech rhythm itself and how it can be measured, with a review of speech rhythm metrics, and suggestions on how these might be improved.

d) Chapter 4 reviews existing research on speech rhythm in varieties of English with a view to evaluating whether Outer Circle varieties tend to be more syllable-timed than Inner Circle ones. There is a criticism that most research does not look at a variety of speaking styles; this is one area Fuchs attempts to address in his research by looking at read speech (a passage) and an interview task.

e) Chapter 5 is a classic methodology section, giving an account of the data used in the study (material and tasks; recording procedure; profile of speakers) and the methods used to annotate and analyse it.

f) Chapter 6 presents the results of Fuchs's production study, with sections on vocalic and consonantal durations, syllable durations, sonority, voicing, fundamental frequency, intensity, loudness, speech rate, and word initial glottal stop insertion before vowels. It is here where he introduces his multidimensional model of speech rhythm.

g) Chapter 7 looks at the perception of IndE and BrE speech rhythm, detailing first pilot studies and then main studies on accent discrimination and 'Cocktail Party Effect', and introducing a "partially new technique" (Fuchs, 2016: 5) for the measurement of speech rhythm perception events.

h) Chapter 8 is a general conclusion, summarising the results, describing the phonology of current IndE, speculating about its future development, and highlighting the importance of the research for work on varieties of English. There is a section on the implications of the research presented here for future work on IndE.

i) Finally, there is an appendix and index. References are included at the end of each chapter and not as a list in the end-matter of the book.

Using Kachru's (1985) circles paradigm, India is described as "a fairly typical Outer Circle country" (Fuchs, 2016: 12), with IndE at Schneider's (2003, 2007) fourth phase, exonormative stabilization. Fuchs explains that it is difficult to estimate the number of languages spoken in India, but comments that acrolectal IndE has "a remarkable degree of uniformity" (Fuchs, 2016: 11) despite the number of first languages (L1s) and the possible variation one might expect this to result in. To put this in perspective, it is noted that $23 \%$ of the population of India "know some English, but only 4\% are fluent" (Fuchs, 2016: 17), and that this $4 \%$ constitutes around 50 million speakers.

A review of existing studies on the phonology of IndE highlights one of the issues of conducting research into Outer Circle varieties, which is the selection of speakers; Sirsa and 
Redford (2013; also cited in Fuchs, 2016) point out that there are likely to be more marked differences with respect to L1 influence in the IndE of speakers educated at what they call regional-medium schools in comparison with those educated in English-medium schools. The 20 Indian university student participants in Fuchs's research were all educated in Englishmedium primary secondary schools with the exception of one, who attended a Telugumedium primary school but an English-medium secondary school, and so this research is very much focussed on speakers who fall into the $4 \%$ fluent, acrolectal group. Fuchs's speakers, who are equally divided between male and female and four L1s (Hindi, Bengali, Telugu and Malayalam), are compared with 10 British male students from the DyVis corpus (Nolan, McDougall, de Jong \& Hudson, 2006).

Fuchs (2016) adopts the terms "stress-timed" and "syllable-timed" throughout the book. There is no discussion of the validity of these terms at any point, although he does mention work by Dauer (1983). While I think it wise to use them (they are useful shorthand for the matter under discussion), I could see criticism might be levelled at the author for not eschewing this controversial terminology—or, at minimum, discussing in more detail the problems associated with it - and simply using the language of the metrics. My feeling is that, in using "stress-timed/syllable-timed" as part of his narrative, Fuchs has made this more accessible to readers from a less phonetics-research-oriented background, such as teachers of English in India and elsewhere who are used to this terminology in pronunciation and general English language teaching text books, and has therefore increased the potential impact of this work outside of academia. Presenting teachers with a Pairwise Variability Index score, for example, usually results in blank looks in my experience (and is, in fact, one of the reasons for adopting a hierarchical research paradigm in my early work on Hong Kong English speech rhythm; see Setter, 2006).

Fuchs's discussion and subsequent use of rhythm metrics in his research leaves us in no doubt, however, that he is fully in control of appropriate research tools. I consider his account and critique of rhythm metrics in Chapter 3 to be one of the best evaluative summaries I have read on the subject, and certainly one of the most accessible. Fuchs makes a valuable contribution to the discussion of the validity and efficacy of metrics in the successful differentiation of speech rhythm in languages, language varieties and types of spoken events. There is extensive review of the work of Arvaniti and colleagues (Arvaniti, 2009, 2012; Arvaniti, Ross \& Ferjan, 2008), for example, and a comparison with research into the variation of vowels to look for insights into how to combat issues in the measurement of speech rhythm.

Fuchs concludes that it is necessary to draw a distinction between "rhythm as a perceptual entity and rhythm as an acoustic entity" (2016: 65) in a similar way to the distinction between pitch, a perceptual event, and fundamental frequency, a production event. Whether there is a direct link between metrics as a measure of the production of speech rhythm and the ability of listeners to categorise speech events into different rhythmic 
categories is one of the things he sets out to test in his research. He then goes on to use a variety of rhythm metrics in his research methodology, justifying his choice of metrics and explaining what he hopes using each one will reveal in his data. Rhythm metrics are not the only measures Fuchs applies to his speech data to investigate rhythm, however. He also incorporates aspects of sonority, differences in intensity and loudness and the insertion of glottal stops before vowels at word boundaries - all of which have been looked at or noted in other studies, but not in combination - into his analysis, to form what he refers to as a "multidimensional model of rhythm" (Fuchs, 2016: 4). This is his real and tangible contribution to research in speech rhythm research.

The methodology sections, as mentioned above, are a study in good practice in methodology procedure and write-up, clear and detailed, and examples which any $\mathrm{PhD}$ student engaged in quantitative research of this kind could benefit from reading. If the PhD write-up itself was akin to the writing in this book, it must have been a delight to examine; I found the whole thing to be a page-turner, and that is not something which is easily said of $\mathrm{PhD}$ theses (or many research journal articles). This does not mean, however, that the writing is over-simplistic or that there is a lack of research rigour.

As I do not want to spoil the readers' enjoyment of this text by giving away the results of the study, in the true spirit of reviewing the page-turner, I will stop here. What I will do is quote the author in his concluding chapter, when he writes that his study makes "a crucial contribution" (Fuchs, 2016: 209) to research in the area of production and perception of speech rhythm. He is not underestimating this contribution, in my opinion.

Discovering that a book with an interesting-looking title is actually someone's $\mathrm{PhD}$ thesis turned into a monograph can lead to mixed reactions. Often, these publications are niche in the extreme and fail to relate the research to the broader subject area in a manner which makes it interesting, accessible and relevant to people working outside that very tiny niche. Fuchs (2016) avoids this admirably. There is plenty in here for the researcher interested in varieties of English, Postcolonial Englishes, Indian English as a variety, speech rhythm, speech rhythm metrics, good practice in the selection and collection of spoken data for instrumental analysis, and how to go about conducting good quality speech research per se; I recommend this as an addition to your personal shelves or university library if you are interested in any of those areas. What's more, it is very well-written in a style which can reach readers outside of a university research audience, should they venture into this area looking for something more focussed than a general book on Indian English such as Sailaja (2009).

\section{REFERENCES}

Arvaniti, A. (2009). Rhythm, timing and the timing of rhythm. Phonetica, 66, 46-63. 
Arvaniti, A. (2012). The usefulness of metrics in the quantification of speech rhythm. Journal of Phonetics, 40, 351-373.

Arvaniti, A., Ross, T. \& Ferjan, N. (2008). On the reliability of rhythm metrics. Journal of the Acoustical Society of America, 124, 2495.

Dauer, R. M. (1983). Stress-timing and syllable-timing reanalyzed. Journal of Phonetics, 11, 51-62.

Kachru, B. B. (1985). Standards, codification and sociolinguistic realism: The English language in the outer circle. In R. Quirk \& H. Widdowson (Eds.), English in the world: Teaching and learning the language and literatures (pp. 11-30). Cambridge: Cambridge University Press.

Nolan, F., McDougall, K., de Jong, G. \& Hudson, T. (2006). A forensic phonetic study of dynamic sources of variability in speech: the DyViS project. In P. Warren \& C. I. Watson (Eds.), Proceedings of the 11th Australasian international conference on speech science and technology (pp. 13-18). Auckland: Australian Speech Science \& Technology Association Inc.

Sailaja, P. (2009). Indian English. Edinburgh: Edinburgh University Press.

Schneider, E. W. (2003). The dynamics of new Englishes: From identity construction to dialect birth. Language, 79, 233-281.

Schneider, E. W. (2007). Postcolonial English: Varieties around the world. Cambridge: Cambridge University Press.

Setter J. (2006). Speech rhythm in world Englishes: The case of Hong Kong. TESOL Quarterly, 40, $763-782$.

Sirsa, H. \& Redford, M. A. (2013). The effects of native language on Indian English sounds and timing patterns. Journal of Phonetics, 41, 393-406. 